\title{
Dynamic modulation of inflammatory pain-related affective and sensory symptoms by optical control of amygdala metabotropic glutamate receptor 4
}

Charleine Zussy ${ }^{1,2}$, Xavier Gómez-Santacana ${ }^{3,4}$, Xavier Rovira ${ }^{1,2}$, Dimitri De Bundel ${ }^{1,2 \dagger}$, Sara Ferrazzo $^{5}$, Daniel Bosch ${ }^{6}$, Douglas Asede ${ }^{6}$, Fanny Malhaire ${ }^{1,2}$, Francine Acher ${ }^{7}$, Jesús Giraldo $^{4,8}$, Emmanuel Valjent ${ }^{1,2}$, Ingrid Ehrlich ${ }^{6}$, Francesco Ferraguti ${ }^{5}$, Jean-Philippe $\operatorname{Pin}^{1,2}$, Amadeu Llebaria $^{3 *}$, Cyril Goudet ${ }^{1,2 *}$

1 Institut de Génomique Fonctionnelle, CNRS, UMR-5203, Université de Montpellier, F34000 Montpellier, France

${ }^{2}$ INSERM, U1191, F-34000 Montpellier, France

${ }^{3}$ MCS, Laboratory of Medicinal Chemistry, Institute for Advanced Chemistry of Catalonia (IQAC-CSIC), Barcelona, Spain

${ }^{4}$ Laboratory of Molecular Neuropharmacology and Bioinformatics, Institut de Neurociències and Unitat de Bioestadística, Universitat Autònoma de Barcelona, 08193 Bellaterra, Spain

${ }^{5}$ Department of Pharmacology, Innsbruck Medical University, Peter Mayr Straße 1a, 6020 Innsbruck, Austria.

${ }^{6}$ Hertie Institute for Clinical Brain Research, University of Tübingen, Otfried-Müller-Straße 25, 72076 Tübingen, Germany; Werner Reichardt Centre for Integrative Neuroscience, University of Tübingen, Otfried-Müller-Straße 25, 72076 Tübingen, Germany.

7 Laboratoire de Chimie et Biochimie Pharmacologiques et Toxicologiques, CNRS UMR8601, Université Paris Descartes, Sorbonne Paris Cité, F-75270 Paris Cedex 6, France

${ }^{8}$ Network Biomedical Research Center on Mental Health (CIBERSAM)

† present address: Department of Pharmaceutical Chemistry and Drug Analysis, Center for Neurosciences, Vrije Universiteit Brussel, 103 Laarbeeklaan, 1090 Brussels, Belgium.

* Corresponding authors: Amadeu Llebaria (amadeu.llebaria@cid.csic.es), Cyril Goudet (cyril.goudet@igf.cnrs.fr) 


\section{ABSTRACT}

Contrary to acute pain, chronic pain does not serve as a warning signal and must be considered as a disease per se. This pathology presents a sensory and psychological dimension at the origin of affective and cognitive disorders. Being largely refractory to current pharmacotherapies, identification of endogenous systems involved in persistent and chronic pain is crucial. The amygdala is a key brain region linking pain sensation with negative emotions. Here, we show that activation of a specific intrinsic neuromodulatory system within the amygdala associated with type 4 metabotropic glutamate receptors $\left(\mathrm{mGlu}_{4}\right)$ abolishes sensory and affective symptoms of persistent pain such as hypersensitivity to pain, anxietyand depression-related behaviors, and fear extinction impairment. Interestingly, neuroanatomical and synaptic analysis of the amygdala circuitry suggests that the effects of mGlu$_{4}$ activation occur outside the central nucleus via modulation of multisensory thalamic inputs to lateral amygdala principal neurons and dorso-medial intercalated cells. Furthermore, we developed optogluram, a small diffusible photoswitchable positive allosteric modulator of $\mathrm{mGlu}_{4}$. This ligand allows the control of endogenous $\mathrm{mGlu}_{4}$ activity with light. Using this optopharmacological approach, we rapidly and reversibly inhibited behavioral symptoms associated with persistent pain through optical control of optogluram in the amygdala of freely behaving animals. Together, our data identifies amygdala $\mathrm{mGlu}_{4}$ signaling as a mechanism that bypasses central sensitization processes to dynamically modulate persistent pain symptoms. Our findings help to define novel and more precise therapeutic interventions for chronic pain, and exemplify the potential of optopharmacology to study the dynamic activity of endogenous neuromodulatory mechanisms in vivo. 


\section{INTRODUCTION}

Chronic pain is a major health problem that affects more than $20 \%$ of the population in Europe and the United States ${ }^{1,2}$, and is poorly alleviated by current treatments ${ }^{3}$. This pathology is characterized by exacerbated responses to both painful (hyperalgesia) and nonpainful (allodynia) stimuli. Besides sensory symptoms, chronic pain is also characterized by impaired emotional responses frequently resulting in anxiety and depression ${ }^{4}$. Glutamate is the main neurotransmitter involved in the transmission of pain-related signals throughout the central nervous system. A loss in the balance between excitatory glutamatergic and inhibitory GABAergic transmission has been suggested to underlie the development of central sensitization which causes the clinical symptoms observed in patients with chronic pain ${ }^{5,6}$.

Metabotropic glutamate receptors (mGluRs) constitute an endogenous modulatory system and are expressed along the entire pain neuraxis where they modulate the perception of pain $^{7}$. We, and others, have previously shown that activation of $\mathrm{mGlu}_{4}$ receptors, which can regulate both glutamate and GABA release at excitatory and inhibitory synaptic terminals ${ }^{7}$, alleviates pain hypersensitivity in preclinical models of chronic pain while leaving acute pain unchanged in naïve animals ${ }^{8-10}$. Moreover, $\mathrm{mGlu}_{4}$ receptors are involved in anxiety and fear processing ${ }^{11,12}$. These properties make $\mathrm{mGlu}_{4}$ receptors an interesting target for new analgesics that are active solely in chronic pain states, and that could impact multiple dimensions of pain.

Growing evidence indicates that the amygdala is one of the key regions for integrating the affective and sensory components of pain ${ }^{13}$. Indeed, the amygdala receives nociceptive inputs through the spinoparabrachial and spinoreticular pathways and modulates pain behavior through projections to descending pain control areas in the brainstem. Chronic pain also induces sensitization in the amygdala, as previously described in several preclinical pain models ${ }^{14,15}$.

Here, we studied whether $\mathrm{mGlu}_{4}$ receptors within the amygdala constitute an intrinsic modulatory system regulating both emotional and sensory dimensions of pain in a murine model of persistent inflammatory pain. To this end, we used behavioral pharmacology and optopharmacology, a novel strategy to manipulate native regulatory mechanisms by light. The use of optogluram, a photoswitchable positive allosteric modulator (PAM) of the $\mathrm{mGlu}_{4}$ receptor, enabled precise and reversible optical control of endogenous mGlu4 activity in the brain of freely behaving mice. Furthermore, we investigated which amygdala pathways are regulated by $\mathrm{mGlu}_{4}$ by means of optogenetic activation of defined pain-related inputs combined with immunoelectron microscopy and whole cell patch clamp recordings. 


\section{MATERIALS AND METHODS}

\section{Ethics}

Animals were treated in accordance with the European Community Council Directive 86/609. Depending on where experiments were performed, experimental protocols were approved by the local authorities (regional animal welfare committee (CEEA-LR) with the guidelines of the French Agriculture and Forestry Ministry (C34-172-13), the Austrian Animal Experimentation Ethics Board, or by the Regierungspraesidium Tuebingen, state of Baden-Wuerttemberg, Germany). All efforts were made to minimize animal suffering and number.

\section{Behavioral studies}

Experiments were performed on 8- to 12-weeks-old C57BL/6J males (Charles River) or $\mathrm{mGlu}_{4} \mathrm{KO}$ mice with wild type (WT) littermates used as controls. Genotyping of $\mathrm{mGlu}_{4} \mathrm{KO}$ mice $^{16}$ was performed as previously described ${ }^{17}$. Persistent inflammatory pain was induced by unilateral intraplantar injection of $30 \mu \mathrm{l}$ complete Freund's adjuvant (CFA; Sigma, Saint Quentin Fallavier, France) in the left hind paw, while control mice received an intraplantar injection of PBS. Guide cannulas were implanted bilaterally by stereotaxic surgery as previously described ${ }^{18}$ and placed over ventro-medial part of the intermediate capsule in the amygdala. For optopharmacology, hybrid cannula combining fluid tubing and an optical fiber were implanted. After 1 week of recovery, animals were subjected to different behavioral tests to assess basal mechanical sensitivity with the von Frey method, fear extinction learning and memory upon auditory cued fear conditioning, anxiety-like behavior in the elevated plus maze (EPM), depressive-like behavior with the splash test, and locomotor activity. Brains were post-fixed to check cannula locations. See supplementary methods for details.

\section{Optopharmacology}

In vitro optopharmacological studies were performed in microplasma free HEK293 cells transiently transfected with rat mGlu receptors by electroporation as previously described ${ }^{19}$. All receptors were cotransfected with the glutamate transporter EAAC1, to preclude interference from extracellular glutamate. Receptors not naturally linked to the phospholipase C (PLC) signaling pathway (Group II and III mGluRs) were cotransfected with a chimeric $\mathrm{Gq} / \mathrm{Gi}$ protein ${ }^{20}$, allowing us to monitor receptor activity through measurements of inositol monophosphate (IP1) production using the IP-One HTRF kit (CisBio Bioassays) ${ }^{21}$. Cells were seeded in a poly-ornithine coated 96-well plate (150000 cells/well), stimulated to induce IP1 accumulation by application of test compounds for 30 minutes at $37^{\circ} \mathrm{C}$, and placed over a LED plate (FCTecnics) for continuous illumination. Fluorescence readings were performed with a RUBYstar microplate reader (BMG Labtech). All measurements were performed in 
triplicate. For selectivity experiments, we measured the effect of optogluram (30 $\mu \mathrm{M})$ on the activation of different mGluRs in the presence of low (EC20) and high (EC80) concentrations of subtype-selective agonists (Quisqualate for group I mGluRs, DCG-IV for group II, and LAP4 for group III mGluRs) to determine potential positive or negative allosteric modulation. In vivo optopharmacological studies were performed for the von Frey test, EPM, and the splash test. We used a compact LED package combining two wavelengths coupled to a rotating optical fiber (fibre diameter: $200 \mu \mathrm{m}, \mathrm{NA}=0.53$ ). Each channel was controlled via the LED driver software (Doric Lenses, Quebec, Canada). Mice were habituated to optic fiber connection one week before the tests. All tests started 20 mins after intra-amygdala injection of optogluram or vehicle. Optical stimulation was delivered using $50 \mathrm{~ms}$ light pulses at $10 \mathrm{~Hz}$ frequency and light powers of $8.0 \mathrm{~mW}$ for $385 \mathrm{~nm}$ wavelength and $2.0 \mathrm{~mW}$ for $505 \mathrm{~nm}$ wavelength. The overall length of light exposure was adapted for each behavioral test. For the von Frey test, mechanical allodynia was tested first in the absence of light stimulation and in the ensuing 25 min in the presence of light stimulation, switching between 385 and $505 \mathrm{~nm}$ wavelength in $5 \mathrm{~min}$ intervals starting with $385 \mathrm{~nm}$. In the EPM, mice were first tested in the absence of light stimulation for $4 \mathrm{~min}$, in the next $4 \mathrm{~min}$ with $385 \mathrm{~nm}$ light stimulation, and the last $4 \mathrm{~min}$ with $505 \mathrm{~nm}$ light stimulation. In the Splash test, grooming behavior was assessed first in the absence of light stimulation for $3 \mathrm{~min}$, in the next $3 \mathrm{~min}$ with $385 \mathrm{~nm}$ light stimulation, and the last 3 min with $505 \mathrm{~nm}$ light stimulation.

\section{Exclusion criteria and group analysis}

Animals were excluded from the study based on pre-established criteria. These were: (1) CFA injection induced $<20 \%$ increase in the response from baseline with the noxious filament, (2) weight loss or prostration behavior occured, that would preclude behavioral analysis, (3) cannulae for intracranial drug delivery were blocked, (4) cannula were incorrectly implanted or removed by the mouse, (5) mice did not learn fear conditioning (less than $20 \%$ freezing to the tone upon memory recall) prior to CFA injection.

The selectivity experiments in vivo on $\mathrm{mGlu}_{4} \mathrm{KO}$ vs. WT mice were performed blind. Some (opto)pharmacological experiments could not be performed blind as (1) several injected compounds were colored, (2) CFA injected mice showed an edema at the paw and (3) wavelength of the delivered light is visible. However, analyses of most behavioral tests were automated to preclude experimenter bias.

\section{Immunocytochemistry for light and electron microscopy}

The distribution of $\mathrm{mGlu}_{4}$ receptors was analyzed using standard light and electron microscopy procedures in fixed brain slices from wt and $\mathrm{mGlu}_{4} \mathrm{KO}$ mouse amygdala. For all details on antibodies and staining procedures, as well as confocal and electron microscopy, see supplementary methods. 


\section{Electrophysiological studies}

Recombinant adeno-associated virus (0.5 $\mathrm{\mu l}$ rAAV-CAG-hChR2(H134R)-mCherry, Penn Vector Core, USA) was injected stereotaxically into the Posterior Intralaminar Nucleus (PIN) and the Medial Geniculate Nucleus (MG) of male GAD67-GFP mice ${ }^{22}$ as previously described $^{28}$. Amygdala slice recordings were performed 4 weeks after injection. Whole-cell recordings were obtained from identified lateral amydala (LA) principal neurons or mediodorsal intercalated cells (mITCd), based on location and the absence or presence of green fluorescence, using 3-5 M $\Omega$ (LA neurons) and 6-8 M $\Omega$ (mITCd cells) borosilicate glass electrodes. Data were acquired with a Multiclamp 700B amplifier, Digidata 1440, and Clampex software (all from MDS, USA). Excitatory postsynaptic currents (EPSCs) were isolated in $100 \mu \mathrm{M}$ Picrotoxin (Sigma, Germany) and evoked either electrically or optically using $470 \mathrm{~nm}$ light pulses $\left(0.2-1 \mathrm{~ms}, 0.5-2 \mathrm{~mW} / \mathrm{mm}^{2}\right)$ from a light emitting diode (LED, CoolLed, UK). All data were analyzed using NeuroMatic (www.neuromatic.thinkrandom.com) and custom-written macros in IgorPro Software (Wavemetrics, USA). For details see supplementary methods.

\section{Ligands and chemicals}

All chemicals were reagent grade (from Roth, Merck, or Sigma, Germany). Quisqualate, DCG-IV, VU0364770 and L-AP4 were purchased from Tocris Bioscience (Bristol, UK). Optogluram [N-(4-((2-chlorophenyl)diazenyl)-3-methoxyphenyl)picolinamide] and LSP4-2022 were synthesized following the experimental procedures previously reported ${ }^{23,24}$.

\section{Statistics}

All data are reported as mean \pm standard error of the mean (SEM), except data from Von Frey experiments which are reported as median \pm interquartile range (IQR). Statistical tests were performed on all datasets as indicated in the Figure legends. Data were analyzed using Prism software (GraphPad, La Jolla, CA, USA) using Student's t-tests (paired or unpaired, two-sided) or analysis of variance (ANOVA) with appropriate post-hoc tests for multiple comparisons as indicated, except data from Von Frey experiments which have been analyzed using non-parametric tests: either the Wilcoxon rank-sum test or the Wilcoxon signed-rank test for unpaired or paired data, respectively , followed by the Holm's method for multiple testing correction. Data were considered significant when $p<0.05$. 


\section{RESULTS}

Sensory and emotional symptoms associated with the CFA-induced inflammatory pain model in mice

We analyzed sensory and emotional components of pain using the CFA-induced inflammatory pain model in mice. Behavioral experiments were performed 8 to 15 days following CFA injection. Interestingly, CFA-induced injury induces symptoms associated with both acute and chronic pain. CFA injection led to mechanical allodynia as revealed by significant increases in paw lifts upon von Frey filament stimulations (Figure 1a, b). When anxiety-like behavior was assessed in the elevated plus maze (EPM), CFA-injected mice spent significantly less time in the open arms (OA) compared to control mice, while no difference was observed in the number of open arm entries (Figure 1c and Supplementary Fig. 1a). Furthermore, CFA-treated mice showed a reduction in the total grooming duration in the splash test when compared to the control group, indicative of a depressive-like behavior (Figure 1d). Importantly, locomotor activity was not significantly altered in CFA-treated mice, allowing us to exclude an indirect effect of persistent pain on locomotion that may confound other behavioral assays (Supplementary Fig. 1b). We further examined whether persistent pain altered fear extinction learning and memory. Classical fear conditioning provides one of the most powerful models to study the neural mechanisms of fear and anxiety ${ }^{13}$. Mice were conditioned just before CFA or vehicle injection. Subsequent group analysis revealed that freezing to the second tone-shock pairing was slightly different, but importantly, both groups reached the same level of freezing at the end of conditioning (Figure 1e). However, the CFAtreated group presented higher freezing levels upon fear memory recall and the ensuing extinction training session, suggesting an increased fear response and an altered acquisition of extinction (Figure 1f). When extinction memory was tested 24 hours later, the conditioned stimulus (CS) again induced higher freezing levels in the CFA group (Figure 19). In summary, our data suggest that the CFA-model recapitulates core symptoms associated with chronic pain such as increased anxiety- and depression-related behavior, and an impaired ability to extinguish fear that may contribute to pathological anxiety often observed in chronic pain patients.

Pharmacological activation of $\mathrm{mGlu}_{4}$ in amygdala abolishes sensory and emotional symptoms associated with CFA-induced injury

Because $\mathrm{mGlu}_{4}$ has been shown to regulate pain and anxiety-related behaviors, we examined the effect of intra-amygdala microinjection of the selective $\mathrm{mGlu}_{4}$ agonist LSP4$2022^{24}(5 \mu \mathrm{M}, 1 \mu \mathrm{L})$ (Supplementary Fig. 2a, b) on allodynia, anxiety- and depression-like behavior and fear extinction in CFA treated mice. First, we observed that the CFA-induced mechanical allodynia was abolished by LSP4-2022 injection (Figure 2a, b and 
Supplementary Fig. 3a, b). Additionally, the enhanced anxiety-like behavior was abolished by $\mathrm{mGlu}_{4}$ activation in the amygdala, as the reduction of OA exploration time in the EPM was abolished by LSP4-2022, but not by vehicle injection in CFA-treated mice (Figure 2c). However, LSP4-2022 injection did not affect the number of OA entries in the EPM or locomotor activity (Supplementary Fig. 3c, d). Depressive-like behavior, as measured by the splash test, was also abolished after the intra-amygdala injection of LSP4-2022, which restored the grooming duration in CFA-treated mice to levels comparable to the control group (Figure 2d). Importantly, we can exclude a non-specific effect of the ligand, as LSP4-2022 at this concentration did not induce any antiallodynic effect in CFA-treated $\mathrm{mGlu}_{4} \mathrm{KO}$ mice (Supplementary Fig. 2c, 4a). To assess the impact of $\mathrm{mGlu}_{4}$ activation on fear and its extinction, we injected mice with LSP4-2022 in the amygdala either before extinction training (Figure 2e, f) or before extinction recall (Supplementary Fig. 3e). Prior to CFA-treatment, fear memory acquisition was similar in both groups (Figure 2e-f, Supplementary Fig. 3e). The experiments with vehicle injection confirmed that fear expression and extinction processes were altered in CFA-treated mice (cf. Figure 1f-g and Supplementary Fig. 3e). Administration of LSP4-2022 before the extinction training improved both extinction learning and extinction recall in CFA-treated mice (Figure 2e), while it had no effect on both processes in control mice (Figure 2f). Conversely, administration of LSP4-2022 before the extinction recall failed to modulate the freezing response (Supplementary Fig. 3e). These data demonstrate that the altered sensory and emotional behaviors observed in our persistent pain model can be suppressed by amygdala activation of $\mathrm{mGlu}_{4}$, and reveal an important role of this receptor in these processes.

\section{$\mathrm{mGlu}_{4}$ is expressed at GABAergic and glutamatergic synapses in the amygdala}

To address where in the pain-related amygdala pathways mGlu $_{4}$ modulates sensory and affective components of pain, we analyzed its distribution at the light and electron microscopy level. Specific immunoreactivity for $\mathrm{mGlu}_{4}$ was mainly present around intercalated cell clusters (ITCs) adjacent to mGlu ${ }_{1 a}$-positive GABAergic projection neurons ${ }^{25}$ and, to a lesser extent, in the neuropil of the LA (Figure 3a-d). In contrast, the central nucleus of the amygdala (CeA), which has been implicated in pain processing and associated with anxiety-like behavioral states ${ }^{26}$, was mostly devoid of specific staining (Figure 3a). Of note, no positive immunosignal for the anti-mGlu $\mathrm{m}_{4}$ antibody was detected in $\mathrm{mGlu}_{4} \mathrm{KO}$ mice (Supplementary Fig. 5a). Using electron microscopy, mGlu $\mathrm{m}_{4}$ was detected at the active zone of presynaptic terminals forming both type I asymmetric (probably glutamatergic) and symmetric (probably GABAergic) synapses ${ }^{27,28}$ in the region surrounding the ITCs (Figure 3e-g). Moreover, $\mathrm{mGlu}_{4}$ was also present in terminals forming type I asymmetric synapses with spines of putative pyramidal neurons in the LA (Figure $\mathbf{3 h}$ ). Using confocal analysis, markers for both GABAergic (vesicular GABA transporter, VGAT) and 
glutamatergic terminals (vesicular glutamate transporter, VGLUT) colocalized with $\mathrm{mGlu}_{4}$ in areas surrounding the ITCs (Figure 3c, d), corroborating our ultrastructural findings. Some of the terminals co-expressed $\mathrm{mGlu}_{4}$ and VGLUT2 (Figure $\mathbf{3 d}$ ), whereas no detectable colocalisation was observed for $\mathrm{mGlu}_{4}$ with VGLUT1 or VGLUT3 (Supplementary Fig. 5b, c).

\section{Presynaptic inhibition of thalamic inputs to mITCd cells and LA principal neurons by $\mathrm{mGlu}_{4}$}

Because of the high $\mathrm{mGlu}_{4}$ mRNA expression in thalamic nuclei ${ }^{29}$ and co-localization with VGLUT2, a marker of thalamic inputs ${ }^{30},{ }^{31}$, we hypothesized that $\mathrm{mGlu}_{4}$ may be involved in gating information from thalamic sensory pathways ${ }^{30}$ to LA and ITCs ${ }^{32-34}$, which is central for emotional processing and fear learning ${ }^{35}$. Therefore, we first analyzed electrically evoked compound thalamic inputs by stimulating the internal capsule while recording excitatory postsynaptic currents (EPSCs) in ( $\mathrm{mlTCd}$ ) cells and LA principal neurons (Figure 4a). Consistent with our anatomical observations, EPSCs were significantly decreased after application of LSP4-2022 (Figure 4d, e). In conjunction, the paired-pulse ratios (as an indicator of presynaptic release probability) were significantly increased after LSP4-2022 application (Figure 4f). In a next step, we labeled and rendered specific projections from the posterior intralaminar nuclei of the thalamus (PIN/MG), that relay multimodal somatosensory and noxious stimuli ${ }^{36}$, light-activatable by viral expression of a Channelrhodopsin2-mCherry fusion protein (Figure 4a-c). Application of LSP4-2022 also significantly decreased the amplitude of optogenetically-activated EPSCs and increased their paired pulse ratios in both mITCd and LA principal neurons (Figure $\mathbf{4 g}$-i). Taken together, our data show that $\mathrm{mGlu}_{4}$ is functionally expressed on specific somatosensory thalamic inputs that converge onto mITCd and LA projections neurons, and that activation of $\mathrm{mGlu}_{4}$ reduces sensory information transfer in this pathway via a presynaptic mechanism.

Particularly the mITCd and mITCv have been implicated in fear extinction ${ }^{37,38}$. Increase in their activation, as measured by expression of the immediate early gene Zif268, accompanies fear extinction learning and recall ${ }^{39}$. Therefore, we asked if basal activation of ITCs was compromised in our pain model. Indeed, we observed a significant reduction in Zif268 expression in ITCs of CFA-treated mice, which was fully rescued by injection of the $\mathrm{mGlu}_{4}$ LSP4-2022 (Supplementary Fig. 6). This experiment was performed 8 days after CFA injection where extinction impairment was observed (Figure 1f-g, 2e-f and Supplementary Fig. 3e). These data suggest that in the persistent inflammatory pain context, $\mathrm{mGlu}_{4}$ activation restores recruitment of ITCs, which could contribute to improving fear extinction learning and memory in CFA-treated mice. 
Optopharmacological manipulation of endogenous amygdala mGlu $\mathrm{u}_{4}$ dynamically regulates sensory and emotional symptoms associated with CFA-induced injury

We employed optopharmacological experiments to address if sensory and emotional behaviors are rapidly and dynamically regulated by endogenous $\mathrm{mGlu}_{4}$ receptors in vivo. We used optogluram, the first photoswitchable PAM of $\mathrm{mGlu}_{4}{ }^{23}$. Optogluram bears an azobenzene photoisomerizable group (Figure 5a) that allows for selective, reversible and repeated optical manipulation of $\mathrm{mGlu}_{4}$ activity with light. Isomerization from trans to cisconfiguration was rapidly achieved upon illumination with violet light $(380 \mathrm{~nm})$ and the transisomer could be recovered from the cis-isomer upon green light illumination (500 nm) in a fast process or by thermal relaxation in the dark, with a half-life of 6.4 minutes (Figure 5b). Both isomers were detected by UV-visible absorption spectroscopy after illumination with either green or violet light (Figure 5c), and photoisomerization was stable and completely reversible upon successive illumination cycles at 380 and $500 \mathrm{~nm}$ (Figure 5d). Optical properties of optogluram enabled the photocontrol of $\mathrm{mGlu}_{4}$ activity in cultured HEK293 cells expressing $\mathrm{mGlu}_{4}$ receptors. Enhancement of $\mathrm{mGlu}_{4}$ activity by optogluram observed in the trans form was reduced upon isomerization to the cis configuration after irradiation with violet light, contrary to the classical mGlu4 PAM VU0364770 ${ }^{40}$ that was insensitive to light (Figure 5e). In vitro, optogluram was selective for $\mathrm{mGlu}_{4}$ and $\mathrm{mGlu}_{6}$ (Supplementary Fig. 7a,b), but $\mathrm{mGlu}_{6}$ expression is restricted to the retina ${ }^{41}$. To assess the effects of optogluram on mechanical sensitivity and anxiety- and depression-like behaviors in vivo, mice were stereotaxically implanted with hybrid optic and fluid cannulas in the amygdala (Figure $\mathbf{5} \mathbf{f}$ and Supplementary Fig. 8). Mechanical allodynia induced by CFA-injection was abolished by intra-amygdala injection of optogluram $(30 \mu \mathrm{M}, 1 \mu \mathrm{L})$ but not by vehicle, reflecting the analgesic effect of this compound (Figure $\mathbf{5 g}$ and Supplementary Fig. 7c, d). Furthermore, violet illumination that generates the inactive cis isomer of optogluram abolished its antiallodynic properties, whereas green light illumination that recovers the active trans isomer reactivated its antiallodynic action and restored normal mechanical sensitivity (Figure $\mathbf{5 g}$ and and Supplementary Fig. 7c, d). Importantly, no antiallodynic effect was observed with optogluram in $\mathrm{mGlu}_{4} \mathrm{KO}$ mice, allowing us to exclude off-target effects at this concentration (Supplementary Fig. 4b). In addition, optogluram injection also did not affect locomotion (Supplementary Fig. 3d). In the EPM test, optogluram increased the time spent in the OA, suggesting an anxiolytic effect. Application of violet light severely reduced OA time, an effect that was partially recovered by green light (Figure 5h, Supplementary Fig. 7e). Lastly, intraamygdala injection of optogluram also reduced the depressive-like behavior of CFA-treated mice measured with the splash test. Optogluram increased the grooming duration in CFAtreated mice, whereas violet light illumination reduced it to the levels observed in vehicletreated mice (Figure 5i, Supplementary Fig. 7f). Importantly, the vehicle group showed no 
significant behavioral changes in any of the different light conditions (Figure $\mathbf{5 g}$-i). Taken together, our findings demonstrate that successive optical activation/inactivation of amygdala $\mathrm{mGlu}_{4}$ produced acute and rapidly reversible analgesic, anxiolytic and anti-depressive effects in mice with persistent inflammatory pain. 


\section{DISCUSSION}

The present work identifies amygdala $\mathrm{mGlu}_{4}$ receptors as key players in controlling sensory and affective symptoms associated with CFA-induced persistent inflammatory pain in mice. We also demonstrate that these lasting sensory and emotional impairments can be rapidly alleviated by manipulation of this specific neuromodulatory system in the amygdala, bypassing central sensitization processes and suggesting it as a potential therapeutic target.

In recent years, optogenetic approaches using exogenous expression of light-sensitive channels have been very powerful tools to analyze the neural circuitry involved in pain ${ }^{42}$ and to dissect the functions of specific amygdala subnuclei and cell types ${ }^{43}$. Optopharmacology (also known as photopharmacology) is a novel light-controlled strategy to manipulate endogenous regulatory mechanisms. Here, we took advantage of optogluram, a photoswitchable $\mathrm{mGlu}_{4}$ ligand in order to control native receptors with light. To our knowledge, this is the first work to establish that optopharmacology with a small diffusible drug-like photoswitchable ligand can be used in vivo to regulate behavior in a disease model. Indeed, optogluram allowed us to control persistent pain-related symptoms in a temporally and spatially restricted manner. Therefore, optopharmacology offers a number of advantages, such as: 1) no need for exogenous viral expression of light-activatable proteins as with optogenetics, 2) improved spatial and temporal control of compound activity compared with conventional pharmacological approaches, and 3) small photoswitchable molecules with amenability to drug development. Given the recent intense efforts to develop photocontrollable ligands for a growing number of other ion channels and receptors ${ }^{23,44-48}$, our work may stimulate their use in other disease models and open avenues for next generation therapeutics.

Our study demonstrates that pharmacological modulation of $\mathrm{mGlu}_{4}$ receptors abolished allodynia, anxiety- and depressive-like behavior, and the impairment of fear extinction. Interestingly, treatment with an $\mathrm{mGlu}_{4}$ agonist before the extinction training was sufficient to rescue fear extinction recall, suggesting that excessive fear can be efficiently suppressed without the need of repetitive treatments. Therefore, pharmacological activation of $\mathrm{mGlu}_{4}$ could be combined with cognitive behavioral therapies in a chronic pain context, when extinction-based exposure therapy is required to treat anxiety-related symptoms ${ }^{49,50}$. Furthermore, the fact that depressive-like behavior was also reduced by amygdala $\mathrm{mGlu}_{4}$ activation in mice with persistent pain suggests $\mathrm{mGlu}_{4}$ agonists possess antidepressant activity. Other studies obtained controversial results showing pro-depressant ${ }^{51}$, antidepressant ${ }^{52}$ or no effects ${ }^{53-55}$ of mGlu $_{4}$ modulation. A possible explanation is that these studies used different administration protocols and were conducted in naive animals, in which we also did not observe any anti-depressant effect. 
At the network level, an important finding is that the primary action of $\mathrm{mGlu}_{4}$ receptors very likely occurs outside of the CeA where, in the canonical view, pain and anxiety modulating effects are expected to occur ${ }^{26}$. In particular, the capsular component of the CeA is known to receive nociceptive information from the spinal cord via the parabrachial area (PB) ${ }^{56}$, a pathway that was recently implicated in fear learning ${ }^{57}$. However, PB neurons lack mGlu4 transcripts whereas thalamic nuclei, including the PIN, were shown to express high mGlu4 mRNA levels ${ }^{29}$. Polymodal sensory information from thalamic and cortical inputs is processed by lateral and basolateral amygdala and ITCs relays ${ }^{58}$. This amygdala network is believed to bring affective valence to sensory information and to play an essential role in anxiety and fear ${ }^{59-61}$. Interestingly, we observed axon terminals containing $\mathrm{mGlu}_{4}$ primarily around ITCs and in the neuropil of the LA. This suggests that $\mathrm{mGlu}_{4}$ receptors modulate polymodal sensory information from the thalamus rather than purely nociceptive information from the PB. Indeed, colocalization of $\mathrm{mGlu}_{4}$ with VGLUT2 and its function in presynaptic modulation of inputs from the PIN onto ITCs and LA neurons suggest a role in gating this major sensory pathway that also has been implicated in fear and extinction learning ${ }^{37}$. This is consistent with our behavioral data demonstrating that $\mathrm{mGlu}_{4}$ modulates both sensory and affective responses.

Previous studies described that another group III mGlu receptor, $\mathrm{mGlu}_{7}$, is also present on thalamic inputs to ITCs and basal amygdala (BLA) ${ }^{25,62}$. Surprisingly, this apparent similar distribution had an opposite effect on pain modulation, as activation of $\mathrm{mGlu}_{7}$ increased mechanical sensitivity and anxiety-like behavior with no effect on chronic pain ${ }^{63}$. Indeed, the striking level of co-labeling of $\mathrm{mGlu}_{4}$ with VGAT in terminals impinging on a subset of neurons encircling the ITCs suggests a possible modulation of inhibitory synapses, but the origin and functional significance of this microcircuit remains to be identified. Chronic inflammatory pain also significantly reduced the basal activation of ITCs as assessed by immediate early gene analysis, which was rescued by mGlu $_{4}$ activation. This suggests an overall disinhibition of the ITC network, likely by multiple impinging mechanisms. As ITCs are important for extinction recall ${ }^{38}$ and become activated during extinction training and recall ${ }^{39}$, restoration of ITC activity by $\mathrm{mGlu}_{4}$ activation may enable a more effective recruitment that, in turn, contributes to the observed improvement in extinction learning and memory. Clearly, further studies aimed at elucidating a causal role of $\mathrm{mGlu}_{4}$ modulation at specific synapses and microcircuits of the amygdala in the reversal of persistent pain symptoms are required.

From a clinical point of view, it is important to stress that, despite the central sensitization processes that occur in this persistent inflammatory pain model, the amygdala appears to retain its ability to rapidly control pain-related behaviors. Indeed, our optopharmacological approach shows that sensory and emotional impairments, even when established for considerable time, can be rapidly alleviated by manipulating $\mathrm{mGlu}_{4}$ receptors in the 
amygdala. However, further studies using different pain models with different etiologies will be needed to better understand the enrolment of these receptors in the regulation of chronic pain states and validate them as therapeutic targets.

In conclusion, our findings demonstrate that acute pharmacological or optopharmacological activation of $\mathrm{mGlu}_{4}$ can rapidly reverse emotional and sensory symptoms of persistent pain despite central sensitization by acting on specific amygdala networks. This work offers a better understanding of the mechanisms underlying negative emotions associated with chronic pain, and opens new avenues for developing innovative therapeutic strategies combining precise pharmacological interventions with extinction-based behavioral therapies for treatment of chronic pain syndromes. 


\section{ACKNOWLEDGMENTS}

We are grateful to Nicola Romanò, Sophie Laffray, Emmanuel Bourinet, André Calas and Etienne Gontier for research assistance and helpful discussions, and Ebba L. Lagerqvist for critical reading of the manuscript. Cell-based pharmacological assays were performed on the ARPEGE (Pharmacology-Screening-Interactome) platform at the Institute de Génomique Fonctionnelle. We acknowledge financial support from the Agence Nationale de la Recherche (ANR-12-NEUR-0003 and ANR-13-BSV1-006 to C.G.), the ERANET Neuron LIGHTPAIN project (to A.L., J.G. and J.-P.P.), the Fundació La Marató de TV3 (110230, 110231, 110232, to J.G., A.L. and C.G.), the Fondation Recherche Médicale (FRM team DEQ20130326522 to J.-P.P), the Centre National de la Recherche Scientifique (F.A., J.-P.P., and C.G.), the Catalan government (2012 BEI_ 00597 to X.G.-S,. and 2014SGR-0109 to A.L.), the Federation of European Biochemical Societies and the Spanish Government (CTQ2014-57020-R to A.L. and SAF2014-58396-R to J.G.), the Beatriu de Pinós program of Agència de Gestió d'Ajuts Universitaris i de Recerca (AGAUR, to X.R.), the Charitable Hertie Foundation (to I.E.), the Werner Reichardt Centre for Integrative Neuroscience at the University of Tuebingen, an Excellence Cluster funded by the Deutsche Forschungsgemeinschaft (DFG, EXC 307, to I.E.), and the Austrian Science Fund (Fonds zur Förderung der Wissenschaftlichen Forschung, Sonderforschungsbereich grant F44-17B23 and W012060-10 to F.F.)

\section{AUTHOR CONTRIBUTION}

C.Z. conceived, performed and analyzed behavioral pharmacology, immunofluorescence microscopy and immediate early gene experiments, and wrote the paper. X.G.S. designed and synthesized optogluram, characterized photoisomerization, and performed and analyzed cell-based pharmacological experiments. X.R. performed and analyzed cell-based pharmacological experiments. D.D.B. performed and analyzed behavioral pharmacology experiments. S.F. performed immunofluorescence and electron microscopy experiments. D.B. and D.A. performed and analyzed classical and optogenetic-based electrophysiological experiments. F.M. performed and analyzed cell-based pharmacological experiments. F.A. designed and synthesized LSP4-2022. J.G. supervised and analyzed pharmacological experiments. E.V. supervised and designed behavioral experiments. I.E. supervised and designed electrophysiology experiments. F.F. supervised and designed neuroanatomy experiments. J.-P.P analyzed pharmacological results, designed experiments and analyzed activity data. A.L. conceived and supervised the project, planned experiments, designed compounds. C.G conceived and supervised the project, designed and analyzed results and wrote the paper. All authors made comments and corrections to the manuscript. 


\section{COMPETING FINANCIAL INTERESTS}

A.L., J.G., X.G.-S, X.R., C.G. and J.-P.P. have filed a patent application for photochromic allosteric modulators of metabotropic glutamate receptors. 


\section{REFERENCES}

1. Breivik H, Collett B, Ventafridda V, Cohen R, Gallacher D. Survey of chronic pain in Europe: prevalence, impact on daily life, and treatment. Eur J Pain 2006; 10(4): 287333.

2. Johannes CB, Le TK, Zhou X, Johnston JA, Dworkin RH. The prevalence of chronic pain in United States adults: results of an Internet-based survey. J Pain 2010; 11(11): 1230-1239.

3. Nightingale S. The neuropathic pain market. Nat Rev Drug Discov 2012; 11(2): 101102.

4. Bushnell MC, Ceko M, Low LA. Cognitive and emotional control of pain and its disruption in chronic pain. Nat Rev Neurosci 2013; 14(7): 502-511.

5. Basbaum Al, Bautista DM, Scherrer G, Julius D. Cellular and molecular mechanisms of pain. Cell 2009; 139(2): 267-284.

6. Latremoliere A, Woolf CJ. Central sensitization: a generator of pain hypersensitivity by central neural plasticity. J Pain 2009; 10(9): 895-926.

7. Goudet C, Magnaghi V, Landry M, Nagy F, Gereau RWt, Pin JP. Metabotropic receptors for glutamate and GABA in pain. Brain Res Rev 2009; 60(1): 43-56.

8. Goudet C, Chapuy E, Alloui A, Acher F, Pin JP, Eschalier A. Group III metabotropic glutamate receptors inhibit hyperalgesia in animal models of inflammation and neuropathic pain. Pain 2008; 137(1): 112-124.

9. Vilar B, Busserolles J, Ling B, Laffray S, Ulmann L, Malhaire F et al. Alleviating pain hypersensitivity through activation of type 4 metabotropic glutamate receptor. $\mathrm{J}$ Neurosci 2013; 33(48): 18951-18965.

10. Wang H, Jiang W, Yang R, Li Y. Spinal metabotropic glutamate receptor 4 is involved in neuropathic pain. Neuroreport 2011; 22(5): 244-248.

11. Davis MJ, Haley T, Duvoisin RM, Raber J. Measures of anxiety, sensorimotor function, and memory in male and female mGluR4(-)/(-) mice. Behav Brain Res 2012; 229(1): 21-28.

12. Davis MJ, lancu OD, Acher FC, Stewart BM, Eiwaz MA, Duvoisin RM et al. Role of mGluR4 in acquisition of fear learning and memory. Neuropharmacology 2013; 66: 365-372.

13. Neugebauer V. Amygdala pain mechanisms. Handb Exp Pharmacol 2015; 227: 261284. 
14. Neugebauer V, Li W, Bird GC, Bhave G, Gereau RWt. Synaptic plasticity in the amygdala in a model of arthritic pain: differential roles of metabotropic glutamate receptors 1 and 5 . J Neurosci 2003; 23(1): 52-63.

15. Jiang $\mathrm{H}$, Fang $\mathrm{D}$, Kong LY, Jin ZR, Cai J, Kang XJ et al. Sensitization of neurons in the central nucleus of the amygdala via the decreased GABAergic inhibition contributes to the development of neuropathic pain-related anxiety-like behaviors in rats. Mol Brain 2014; 7: 72.

16. Pekhletski R, Gerlai R, Overstreet LS, Huang XP, Agopyan N, Slater NT et al. Impaired cerebellar synaptic plasticity and motor performance in mice lacking the mGluR4 subtype of metabotropic glutamate receptor. J Neurosci 1996; 16(20): 63646373.

17. Pitsch J, Schoch S, Gueler N, Flor PJ, van der Putten H, Becker AJ. Functional role of mGluR1 and mGluR4 in pilocarpine-induced temporal lobe epilepsy. Neurobiol Dis 2007; 26(3): 623-633.

18. De Bundel D, Zussy C, Espallergues J, Gerfen CR, Girault JA, Valjent E. Dopamine D2 receptors gate generalization of conditioned threat responses through mTORC1 signaling in the extended amygdala. Mol Psychiatry 2016.

19. Brabet I, Parmentier ML, De Colle C, Bockaert J, Acher F, Pin JP. Comparative effect of L-CCG-I, DCG-IV and gamma-carboxy-L-glutamate on all cloned metabotropic glutamate receptor subtypes. Neuropharmacology 1998; 37(8): 1043-1051.

20. Gomeza J, Mary S, Brabet I, Parmentier ML, Restituito S, Bockaert J et al. Coupling of metabotropic glutamate receptors 2 and 4 to $\mathrm{G}$ alpha $15, \mathrm{G}$ alpha 16 , and chimeric $\mathrm{G}$ alpha q/i proteins: characterization of new antagonists. Mol Pharmacol 1996; 50(4): 923-930.

21. Trinquet E, Fink M, Bazin H, Grillet F, Maurin F, Bourrier E et al. D-myo-inositol 1phosphate as a surrogate of $\mathrm{D}$-myo-inositol 1,4,5-tris phosphate to monitor $\mathrm{G}$ proteincoupled receptor activation. Anal Biochem 2006; 358(1): 126-135.

22. Tamamaki N, Yanagawa Y, Tomioka R, Miyazaki J, Obata K, Kaneko T. Green fluorescent protein expression and colocalization with calretinin, parvalbumin, and somatostatin in the GAD67-GFP knock-in mouse. J Comp Neurol 2003; 467(1): 6079.

23. Pittolo S, Gomez-Santacana X, Eckelt K, Rovira X, Dalton J, Goudet C et al. An allosteric modulator to control endogenous $\mathrm{G}$ protein-coupled receptors with light. Nat Chem Biol 2014; 10(10): 813-815.

24. Goudet C, Vilar B, Courtiol T, Deltheil T, Bessiron T, Brabet I et al. A novel selective metabotropic glutamate receptor 4 agonist reveals new possibilities for developing subtype selective ligands with therapeutic potential. FASEB J 2012; 26(4): 16821693. 
25. Bienvenu TC, Busti D, Micklem BR, Mansouri M, Magill PJ, Ferraguti F et al. Large intercalated neurons of amygdala relay noxious sensory information. J Neurosci 2015; 35(5): 2044-2057.

26. Veinante $\mathrm{P}$, Yalcin I, Barrot $\mathrm{M}$. The amygdala between sensation and affect: a role in pain. J Mol Psychiatry 2013; 1(1): 9.

27. Gray EG. Axo-somatic and axo-dendritic synapses of the cerebral cortex: an electron microscope study. J Anat 1959; 93: 420-433.

28. Harris KM, Weinberg RJ. Ultrastructure of synapses in the mammalian brain. Cold Spring Harb Perspect Biol 2012; 4(5).

29. Ohishi H, Akazawa C, Shigemoto R, Nakanishi S, Mizuno N. Distributions of the mRNAs for L-2-amino-4-phosphonobutyrate-sensitive metabotropic glutamate receptors, mGluR4 and mGluR7, in the rat brain. J Comp Neurol 1995; 360(4): 555570.

30. Fremeau RT, Jr., Voglmaier S, Seal RP, Edwards RH. VGLUTs define subsets of excitatory neurons and suggest novel roles for glutamate. Trends Neurosci 2004; 27(2): 98-103.

31. Barroso-Chinea $P$, Castle M, Aymerich MS, Lanciego JL. Expression of vesicular glutamate transporters 1 and 2 in the cells of origin of the rat thalamostriatal pathway. J Chem Neuroanat 2008; 35(1): 101-107.

32. Asede D, Bosch D, Luthi A, Ferraguti F, Ehrlich I. Sensory inputs to intercalated cells provide fear-learning modulated inhibition to the basolateral amygdala. Neuron 2015; 86(2): 541-554.

33. Sigurdsson T, Doyere V, Cain CK, LeDoux JE. Long-term potentiation in the amygdala: a cellular mechanism of fear learning and memory. Neuropharmacology 2007; 52(1): 215-227.

34. Sah P, Lopez De Armentia M. Excitatory synaptic transmission in the lateral and central amygdala. Ann N Y Acad Sci 2003; 985: 67-77.

35. Pape HC, Pare D. Plastic synaptic networks of the amygdala for the acquisition, expression, and extinction of conditioned fear. Physiol Rev 2010; 90(2): 419-463.

36. Lanuza E, Nader K, Ledoux JE. Unconditioned stimulus pathways to the amygdala: effects of posterior thalamic and cortical lesions on fear conditioning. Neuroscience 2004; 125(2): 305-315.

37. Duvarci S, Pare D. Amygdala microcircuits controlling learned fear. Neuron 2014; 82(5): 966-980. 
38. Likhtik E, Popa D, Apergis-Schoute J, Fidacaro GA, Pare D. Amygdala intercalated neurons are required for expression of fear extinction. Nature 2008; 454(7204): 642645.

39. Busti D, Geracitano R, Whittle N, Dalezios Y, Manko M, Kaufmann W et al. Different fear states engage distinct networks within the intercalated cell clusters of the amygdala. J Neurosci 2011; 31(13): 5131-5144.

40. Jones CK, Bubser M, Thompson AD, Dickerson JW, Turle-Lorenzo N, Amalric M et al. The metabotropic glutamate receptor 4-positive allosteric modulator VU0364770 produces efficacy alone and in combination with L-DOPA or an adenosine $2 \mathrm{~A}$ antagonist in preclinical rodent models of Parkinson's disease. J Pharmacol Exp Ther 2012; 340(2): 404-421.

41. Nakajima $\mathrm{Y}$, Iwakabe $\mathrm{H}$, Akazawa $\mathrm{C}$, Nawa $\mathrm{H}$, Shigemoto $\mathrm{R}$, Mizuno $\mathrm{N}$ et al. Molecular characterization of a novel retinal metabotropic glutamate receptor mGluR6 with a high agonist selectivity for L-2-amino-4-phosphonobutyrate. J Biol Chem 1993; 268(16): 11868-11873.

42. Carr FB, Zachariou V. Nociception and pain: lessons from optogenetics. Front Behav Neurosci 2014; 8: 69.

43. Lalumiere RT. Optogenetic dissection of amygdala functioning. Front Behav Neurosci 2014; 8: 107.

44. Bahamonde MI, Taura J, Paoletta S, Gakh AA, Chakraborty S, Hernando J et al. Photomodulation of $\mathrm{G}$ protein-coupled adenosine receptors by a novel lightswitchable ligand. Bioconjug Chem 2014; 25(10): 1847-1854.

45. Broichhagen J, Schonberger M, Cork SC, Frank JA, Marchetti P, Bugliani M et al. Optical control of insulin release using a photoswitchable sulfonylurea. Nat Commun 2014; 5: 5116.

46. Kokel D, Cheung CY, Mills R, Coutinho-Budd J, Huang L, Setola V et al. Photochemical activation of TRPA1 channels in neurons and animals. Nat Chem Biol 2013; 9(4): 257-263.

47. Schonberger M, Trauner D. A photochromic agonist for mu-opioid receptors. Angew Chem Int Ed Engl 2014; 53(12): 3264-3267.

48. Stein M, Middendorp SJ, Carta V, Pejo E, Raines DE, Forman SA et al. Azopropofols: photochromic potentiators of $\mathrm{GABA}(\mathrm{A})$ receptors. Angew Chem Int Ed Engl 2012; 51(42): 10500-10504.

49. Mahan AL, Ressler KJ. Fear conditioning, synaptic plasticity and the amygdala: implications for posttraumatic stress disorder. Trends Neurosci 2012; 35(1): 24-35. 
50. Herry C, Ferraguti F, Singewald N, Letzkus JJ, Ehrlich I, Luthi A. Neuronal circuits of fear extinction. Eur J Neurosci 2010; 31(4): 599-612.

51. Podkowa K, Rzezniczek S, Marciniak M, Acher F, Pilc A, Palucha-Poniewiera A. A novel mGlu4 selective agonist LSP4-2022 increases behavioral despair in mouse models of antidepressant action. Neuropharmacology 2015; 97: 338-345.

52. Kalinichev M, Le Poul E, Bolea C, Girard F, Campo B, Fonsi M et al. Characterization of the novel positive allosteric modulator of the metabotropic glutamate receptor 4 ADX88178 in rodent models of neuropsychiatric disorders. J Pharmacol Exp Ther 2014; 350(3): 495-505.

53. Klak K, Palucha A, Branski P, Sowa M, Pilc A. Combined administration of PHCCC, a positive allosteric modulator of mGlu4 receptors and ACPT-I, mGlu III receptor agonist evokes antidepressant-like effects in rats. Amino Acids 2007; 32(2): 169-172.

54. Slawinska A, Wieronska JM, Stachowicz K, Palucha-Poniewiera A, Uberti MA, Bacolod MA et al. Anxiolytic- but not antidepressant-like activity of Lu AF21934, a novel, selective positive allosteric modulator of the mGlu(4) receptor. Neuropharmacology 2013; 66: 225-235.

55. Wieronska JM, Stachowicz K, Palucha-Poniewiera A, Acher F, Branski P, Pilc A. Metabotropic glutamate receptor 4 novel agonist LSP1-2111 with anxiolytic, but not antidepressant-like activity, mediated by serotonergic and GABAergic systems. Neuropharmacology 2010; 59(7-8): 627-634.

56. Todd AJ. Neuronal circuitry for pain processing in the dorsal horn. Nat Rev Neurosci 2010; 11(12): 823-836.

57. Han S, Soleiman MT, Soden ME, Zweifel LS, Palmiter RD. Elucidating an Affective Pain Circuit that Creates a Threat Memory. Cell 2015; 162(2): 363-374.

58. Neugebauer V, Galhardo V, Maione S, Mackey SC. Forebrain pain mechanisms. Brain Res Rev 2009; 60(1): 226-242.

59. Pare D, Quirk GJ, Ledoux JE. New vistas on amygdala networks in conditioned fear. J Neurophysiol 2004; 92(1): 1-9.

60. Maren S. Synaptic mechanisms of associative memory in the amygdala. Neuron 2005; 47(6): 783-786.

61. Phelps EA, LeDoux JE. Contributions of the amygdala to emotion processing: from animal models to human behavior. Neuron 2005; 48(2): 175-187.

62. Dobi A, Sartori SB, Busti D, Van der Putten H, Singewald N, Shigemoto R et al. Neural substrates for the distinct effects of presynaptic group III metabotropic 
glutamate receptors on extinction of contextual fear conditioning in mice. Neuropharmacology 2013; 66: 274-289.

63. Palazzo E, Fu Y, Ji G, Maione S, Neugebauer V. Group III mGluR7 and mGluR8 in the amygdala differentially modulate nocifensive and affective pain behaviors. Neuropharmacology 2008; 55(4): 537-545. 


\section{Figure legends}

Figure 1: Inflammatory pain model induced by complete Freund's adjuvant (CFA) recapitulates sensory and emotional symptoms of chronic pain. a, Experimental design and timeline of behavioral tests performed in CFA-treated (CFA) and PBS injected (Control) mice. b. Mechanical allodynia was observed in CFA mice as seen by significant increases in paw lifts upon innocuous, intermediate and noxious von Frey filament stimulation 10 days after injection (CFA, $n=7$ vs. control, $n=10$, nonparametric Wilcoxon rank-sum test $\left.{ }^{* *} p<0.001\right)$. c, Anxiety-like behavior assessed in the elevated plus maze (EPM) revealed a significant decrease in time spent in open arms $(O A)$ in CFA $(n=10)$ vs. control mice $(n=8)$, unpaired t-test, ${ }^{* *} p<0.01$. d, Depressive-like behavior assessed in the splash test revealed a significant decrease in grooming duration in CFA $(n=9)$ vs. control mice $(n=8)$, unpaired ttest, ${ }^{* * *} p<0.001$. e-g Fear conditioning and extinction in CFA $(n=10)$ and control $(n=8)$ mice. e, Fear increased in both groups during conditioning (2-way ANOVA, tone $p<0.0001$, group $p=0.0495$ ), but was not different for the last tone prior to paw injection (Bonferroni-corrected post-hoc tests). f, During extinction learning, freezing decreased in both groups, and CFA mice showed enhanced CS-evoked freezing compared to controls (2-way ANOVA: tone block $p<0.0001$, group $p=0.0038$ ). Analysis of fear recall (tone block 1 ) and end of extinction training (tone block 10) revealed significant extinction, and a difference in freezing at the end of extinction training in control vs. CFA mice (2-way ANOVA: tone block $p<0.0001$, group $p=0.015$; and Bonferroni- corrected post-hoc test of block 10: $p<0.01) . \mathbf{g}$. At extinction recall, CS-evoked freezing was enhanced in CFA mice compared to controls (unpaired t-test, unequal variance, $\left.{ }^{*} p=0.0257\right) .{ }^{*} p<0.05,{ }^{* *} p<0.01$ shown only for Bonferroni-corrected posthoc tests. For extinction learning and recall, data are presented as blocks of $4 \mathrm{CSs}$. All data are expressed as means \pm SEM, except in (b) expressed as median $\pm I Q R$.

Figure 2: Activation of amygdala $\mathrm{mGlu}_{4}$ relieves sensory and emotional symptoms in CFA-treated mice. a, Experimental design and timeline of behavioral tests. Mice received intra-amygdala injections of $1 \mu \mathrm{L}$ LSP4-2022 (5 $\mu \mathrm{M})$ or vehicle (PBS) 20 min before each test. b, Mechanical sensitivity was restored in CFA mice treated with LSP4-2022 as assessed by noxious von Frey filament stimulation 10 days after injection (T0 vs Naïve, T20, T40 and T60 is compared for each group, Ctl-LSP4-2022, n=7; Ctl-vehicle, n=7; CFA-LSP42022, $n=9$ and CFA-vehicle, $n=7,{ }^{*} p<0.05$, nonparametric Wilcoxon signed-rank test, followed by the Holm's method for multiple testing correction). Naive indicates responses of mice before CFA-treatment. c, Anxiety-like behavior induced by CFA injection was reduced by LSP4-2022 treatment as assessed in the EPM (Ctl-LSP4-2022, $n=7$ vs. Ctl-vehicle, $n=10$; CFA-LSP4-2022, $n=11$ vs. CFA-vehicle, $n=8$; unpaired t-test, " $p<0.05$,). d, Depressive-like behavior induced by CFA injection was reduced by LSP4-2022 treatment as assessed in the splash test (Ctl-LSP4-2022, $n=8$ vs. Ctl-vehicle, $n=8$; CFA-LSP4-2022, $n=9$ vs. CFA-vehicle, 
$\mathrm{n}=9$, unpaired t-test, ${ }^{* *} \mathrm{p}<0.001$ ). e, (top) Experimental design and timeline for fear and extinction learning and extinction recall. e-f, (left) Freezing increased during fear conditioning, but was not different between the respective control or CFA groups prior to paw and amygdala injections (CFA-LSP4-2022 n=9 vs. CFA-vehicle, $n=14$; CtI-LSP4-2022, $n=10$ vs. Ctl-vehicle, $n=20$; two-way ANOVAs, tone block <0.0001). e, (middle, right) During extinction learning, CFA mice maintained high freezing levels when treated with vehicle, but reduced freezing when treated with LSP4-2022 (CFA-LSP4-2022, $n=9$ vs. CFA-vehicle, $\mathrm{n}=14$; two-way ANOVA of fear recall (tone block 1) and end of extinction (tone block 9 ): tone block $p=0.0001$, interaction tone block $x$ treatment $p=0.026$; Bonferroni corrected post-hoc tests: CFA-LSP4-2022 tone block 1 vs. block 9: $p<0.001$, and CFA-LSP4-2022 vs. CFAvehicle at tone block 9: $p<0.05)$. At extinction recall, freezing remained decreased in CFALSP4-2022 mice $(n=8)$ vs. CFA-vehicle mice $\left(n=8\right.$, unpaired t-test, $\left.{ }^{* *} p=0.007\right)$. $f$, (middle, right) Both control groups showed significant within session extinction (two-way ANOVA, tone block $p<0.0001$ ), and injection of LSP4-2022 did not affect freezing in control mice (CtlLSP4-2022, $n=11$ vs. Ctl-vehicle, $n=20$; two-way ANOVA fear recall (tone block 1 ) and end of extinction (tone block 9): tone block $p=0.0001$, treatment n.s.). Freezing levels during extinction recall were similar in both groups (Ctl-LSP4-2022, $n=10$ vs. to Ctl-vehicle, $n=14$, unpaired t-test, $p=0.625)$. ${ }^{*} p<0.05,{ }^{* *} p<0.01,{ }^{* * *} p<0.001$ shown only for Bonferroni-corrected post-hoc tests. For extinction learning and recall, data are presented as blocks of 4 CSs. All data are expressed as means \pm SEM, except in (b) where they are expressed as median \pm IQR.

Figure 3: $\mathrm{mGlu}_{4}$ is expressed at GABAergic and glutamatergic synapses in the mouse amygdala. a, Intense $\mathrm{mGlu}_{4}$ immunoreactive puncta decorate the soma and dendrites of neurons neighboring the ITC clusters. Moderate staining is also observed within the basolateral complex and in particular in the LA. Scale bars: $500 \mu \mathrm{m}$. b, Huygensdeconvoluted confocal stack (z-stack: $14.75 \mu \mathrm{m}$, z-step size $0.13 \mu \mathrm{m}$ ) showing $\mathrm{mGlu}_{4}$ (red) immunopositive axon terminals innervating the soma and dendrites of weakly labeled, but not of strongly labeled (*) mGlu1a (green) neurons surrounding the mITCV. Scale bar: $5 \mu \mathrm{m}$. c, d, Co-staining of $\mathrm{mGlu}_{4}$ (red) with the synaptic markers (green) in (c) VGAT and (d) VGLUT 2. Arrowheads indicate colocalisation, note the preferential colocalisation of $\mathrm{mGlu}_{4}$ with VGAT in areas surrounding the mITCv. Scale bars: $10 \mu \mathrm{m}$. e, Electron micrograph of a dendrite (d) located near the mITCv receiveing several axon terminals containing mGlu $_{4}$ (painted in green), which form symmetric (putative GABAergic) synapses. $\mathrm{mGlu}_{4}$ immunoreactivity was visualized by a HRP-DAB reaction resulting in a diffuse electrondense product. Enlarged view of the terminal demarcated by solid lines is shown in the lower panel. Scale bar: $1 \mu \mathrm{m}$. $\mathbf{f}-\mathbf{h}, \mathrm{mGlu}_{4}$ receptors accumulate at the active zone of axon terminals (at), as visualized by silver-intensified nanogold particles, forming both (f) symmetric and $(\mathbf{g})$ asymmetric synapses with (f) dendritic shafts or $(\mathbf{g})$ spines (sp) of neurons surrounding the mITCv. Scale bars: 500 
$\mathrm{nm}$. $\mathbf{h}$, Presynaptic $\mathrm{mGlu}_{4}$ labeled terminal forming an asymmetric synapse with the spine of a putative pyramidal neuron in the LA. Scale bar: $500 \mathrm{~nm}$. Abbreviations: CeA, central amygdala; LA, lateral amygdala; BA, basal amygdala; mITCd, medial dorsal intercalated cell cluster; mITCv, medial ventral ITC.

Figure 4: Presynaptic inhibition of thalamic inputs to dorso-medial ITC (mITCd) cells and lateral amygdala (LA) principal neurons by mGlu4. a, Scheme depicting experimental setup for electrical and optical stimulation of thalamic inputs. b, c, representative confocal images of the thalamic injection site in PIN/MG (b) and corresponding afferent fibers in the amygdala (c) of a GAD67-GFP transgenic mouse. Neurons and fibers expressing ChR-mCherry fusion protein (red), interneurons (green), Neurotrace (blue) was used as a cell marker. Scale bars: 250 and $200 \mu \mathrm{m}$. d, Example traces of EPSCs in mITCd and LA principal neurons evoked by electrical thalamic fiber stimulation during baseline and after application of LSP4-2022 (5 $\mu \mathrm{M})$. e, Summary plot of relative EPSC amplitudes revealing significant reductions in electrically evoked inputs after LSP4-2022 application (mITCd: $78.1 \pm 3.7 \%, n=17$; LA: $60.8 \pm 3.7 \%, n=10$ ). f, Summary plot of relative paired-pulse ratios of electrically evoked inputs revealing significant increases after LSP4-2022 application (mITCd: $118.2 \pm 6.7 \%, n=17$; LA: $112.8 \pm 3.7 \%, n=10)$. g, Example traces of EPSCs in mITCd and LA principal neurons evoked by optical stimulation of thalamic fibers from PIN/MG during baseline and after application of LSP4-2022. $\mathbf{h}$, Summary plot of relative EPSC amplitudes revealing significant reductions in optically evoked inputs after LSP4-2022 application (mITCd: $86.2 \pm 2.6 \%, n=15$; LA: $61.8 \pm 4.2 \%$, $n=10)$. i, Summary plots of relative paired-pulse ratios of optically evoked inputs revealing significant increases after LSP4-2022 application (mITCd opto: $112.1 \pm 4.8 \%, n=15$; LA opto: $123.9 \pm 5.7 \%, n=10$ ). Scale bar for $\mathbf{d}$ and $\mathbf{g}: 100 \mathrm{pA}, 25 \mathrm{~ms}$. All statistical analysis used paired t-tests (baseline vs. LSP4-2022), ${ }^{*} p<0.05,{ }^{* *} p<0.01,{ }^{* * *} p<0.001$.

Figure 5: Dynamic regulation of sensory and emotional symptoms in CFA-treated mice by photoswitchable activation $\mathrm{mGlu}_{4}$ in the amygdala. a, Structure and photoisomerization properties of the $\mathrm{mGlu}_{4}$ PAM optogluram from trans to cis configuration after illumination with violet light $(\lambda=380 \mathrm{~nm})$ and from cis to trans with green light $(\lambda=500$ $\mathrm{nm}$ ). $\mathbf{b}$, Thermal relaxation of cis-optogluram in aqueous solution (30 $\mu \mathrm{M}$ in PBS with $0.3 \%$ DMSO) after 3 min illumination with violet light. An exponential decay function was used to estimate relaxation half-life $\left(t_{1 / 2}=6.4 \mathrm{~min}\right)$. Absorbance is shown as arbitrary units $(A U)$. $\mathbf{c}$, UV-visible absorption spectra of optogluram (30 $\mu \mathrm{M}$ in PBS with $0.3 \%$ DMSO) after 3 min illumination with either green or violet light. $\mathbf{d}$, Reversibility and stability of photoisomerization of optogluram ( $30 \mu \mathrm{M}$ in PBS with $0.3 \%$ DMSO) after 3 min of illumination cycles of with 
violet and green light. e, Light-dependency of optogluram enhancing activity in a cell-based pharmacology assay. Dose-dependent enhancing activity of optogluram and VU0364770 (a conventional mGlu4 PAM) on a nominal concentration of agonist (L-AP4, 3 nM) in dark conditions or under constant illumination with violet light (380 nm). Receptor activation was measured by IP accumulation assay in $\mathrm{mGlu}_{4}$-transfected HEK293 cells stimulated with a constant concentration of L-AP4 in presence of increasing concentrations of PAMs. Potency of optogluram was shifted from $0.60 \pm 0.01 \mu \mathrm{M}(\mathrm{n}=3)$ in dark conditions to $1.93 \pm 0.05 \mu \mathrm{M}(\mathrm{n}=3)$ under violet light, contrary to that of VU0364770 which is insensitive to light. $\mathbf{f}$, Diagram illustrating placement and design of the hybrid cannula for light and fluid delivery in the amygdala. g, Mechanical sensitivity assessed by the noxious von Frey filament was restored in CFA mice treated with optogluram $(30 \mu \mathrm{M})$. The analgesic effect was abolished when optogluram was inactivated by violet light (violet rectangle) and recovered when optogluram was reactivated by green light (green rectangle) (T0 vs Naïve, T20, T25, T30, T35, T40 and T45 for is compared for each group, CFA-optogluram, $n=8{ }^{*} p<0.05$ and CFA-vehicle, $n=9$, $\# p<0.05$, nonparametric Wilcoxon signed-rank test, followed by the Holm's method for multiple testing correction). $\mathbf{h}$, Anxiety-like behavior assessed in the EPM revealed an anxiolytic effect of optogluram $(30 \mu \mathrm{M})$. The optogluram-induced increased open arm exploration was abolished by violet light and increased again by green light. Within group comparisons revealed a significant effect of light in the optogluram-, but not the vehicleinjected group (CFA-optogluram, $n=7$; CFA-vehicle, $n=7$; one-way ANOVA followed by Tukey's post-hoc tests, $\left.{ }^{* *} p<0.01\right)$. i, Depressive-like behavior assessed in the splash test revealed an anti-depressant effect of optogluram $(30 \mu \mathrm{M})$. The optogluram-induced increased grooming duration was abolished by violet light and increased again by green light. Within group comparisons revealed a significant effect of light in the optogluram-, but not the vehicle-injected group (CFA-optogluram, $n=10$; CFA-vehicle, $n=10$; one-way ANOVA followed by Tukey's post-hoc tests, $\left.{ }^{* *} p<0.01\right)$. All data are expressed as means \pm SEM, except in $(\mathbf{g})$ where they are expressed as median \pm IQR. 
a
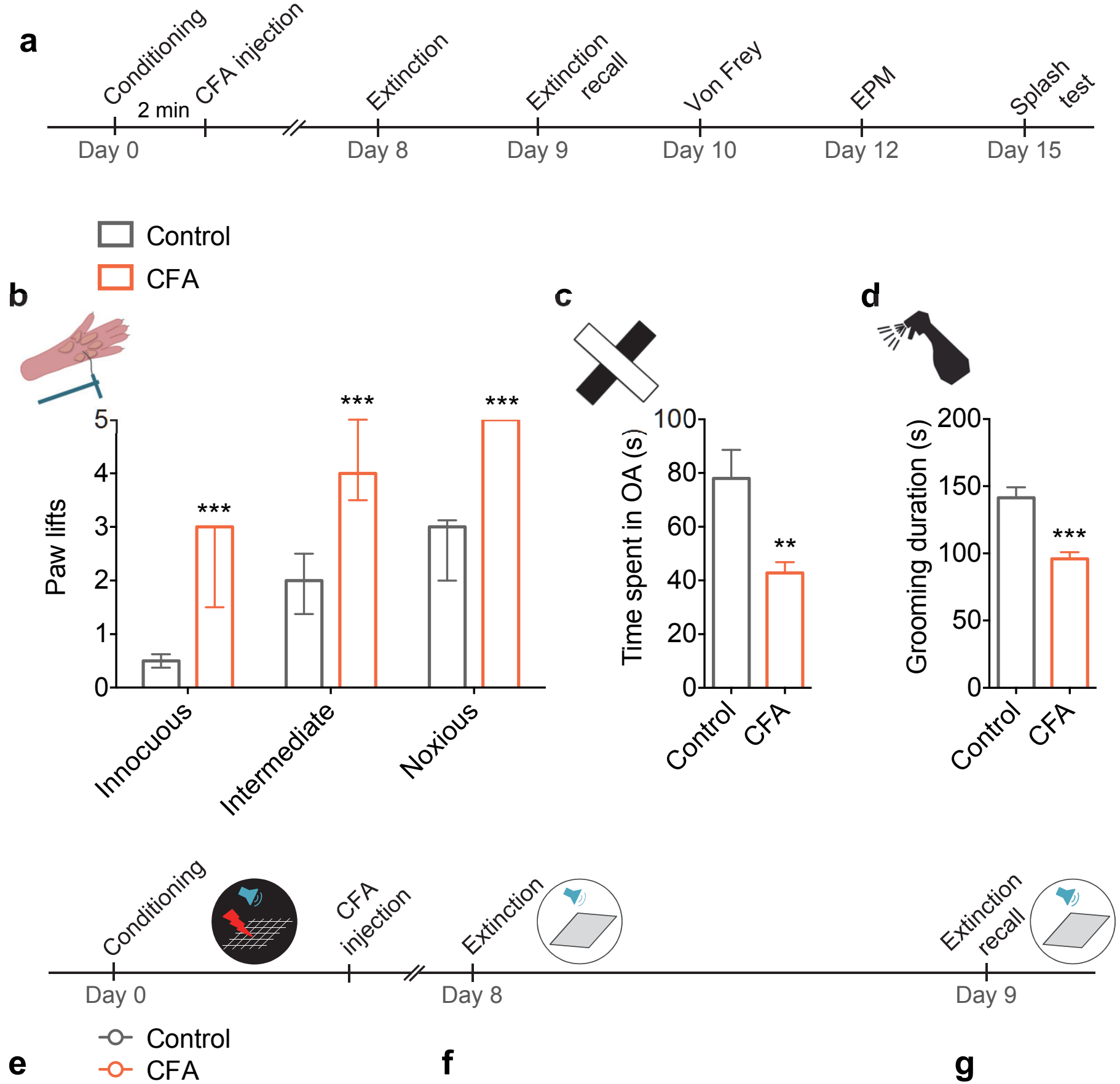

f

g
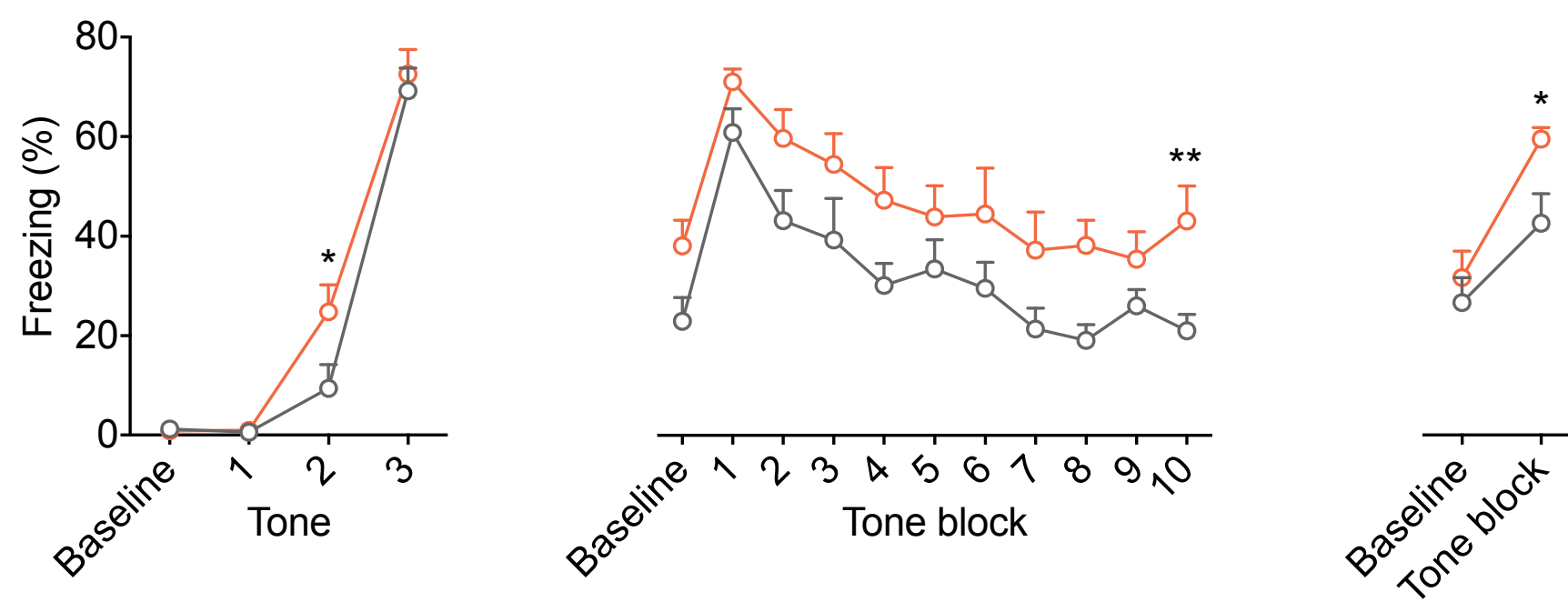

Figure 1 
a
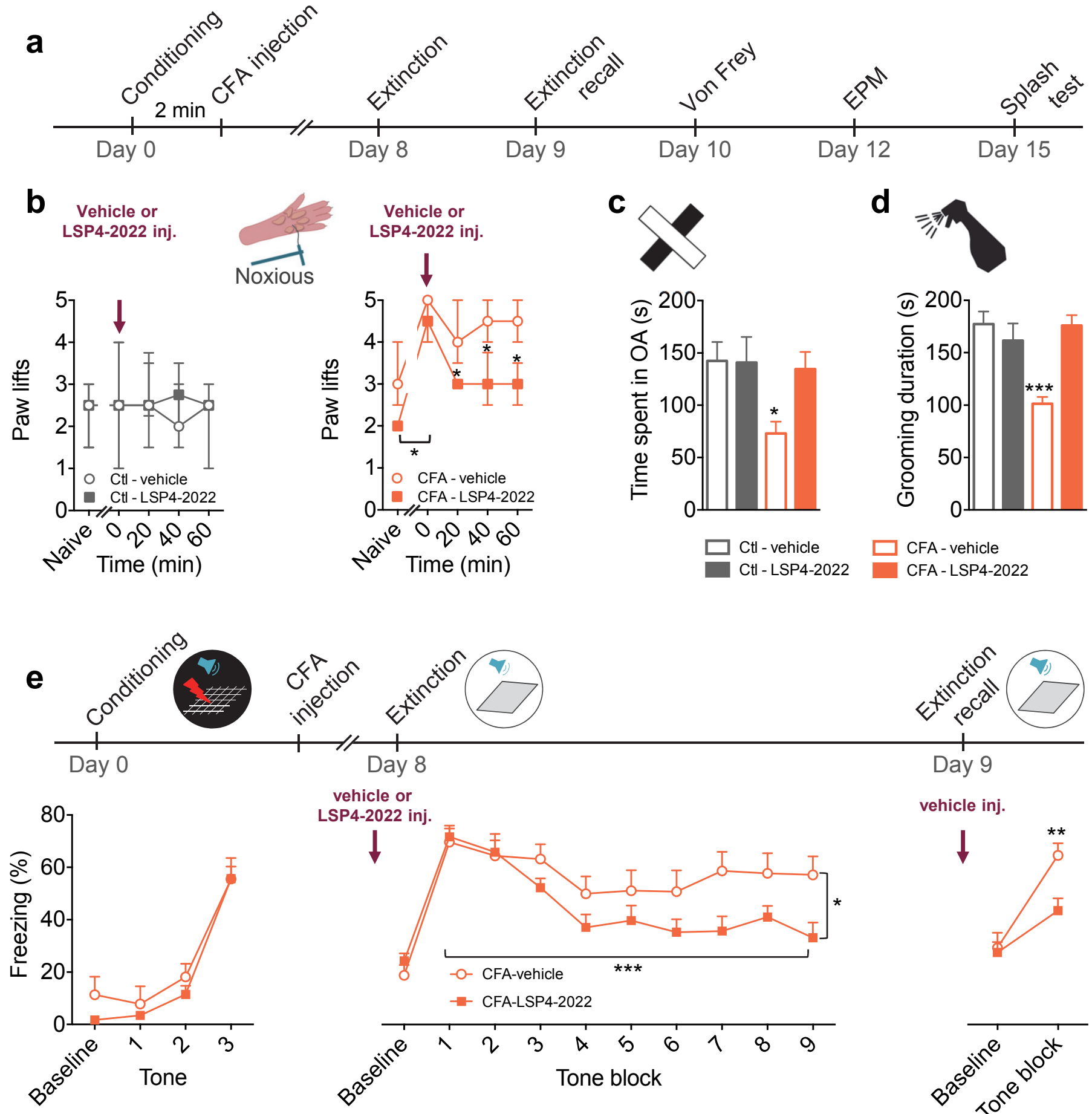

vehicle or
LSP4-2022 inj.

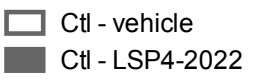

CFA - vehicle

CFA - LSP4-2022
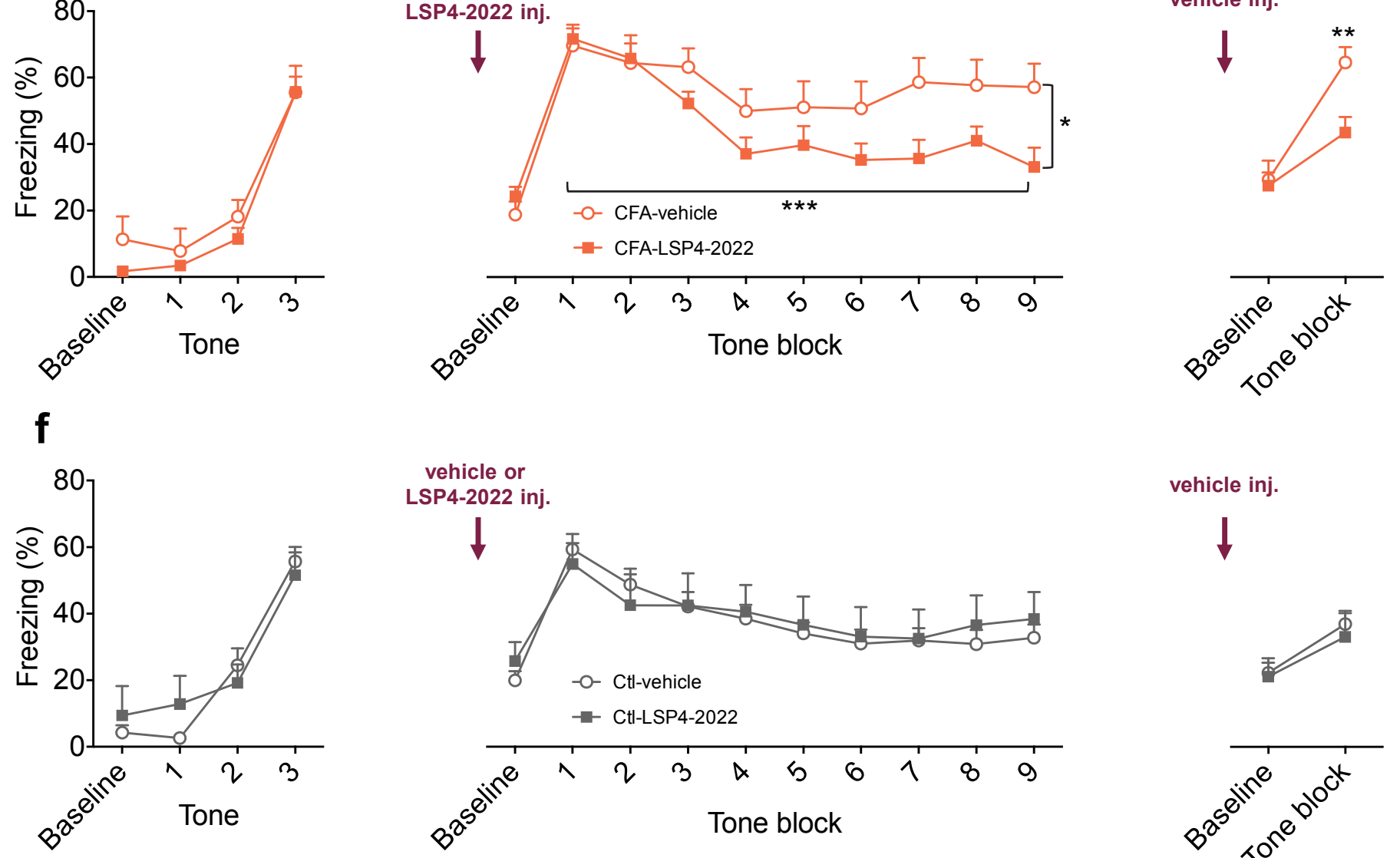

vehicle or

LSP4-2022 inj.

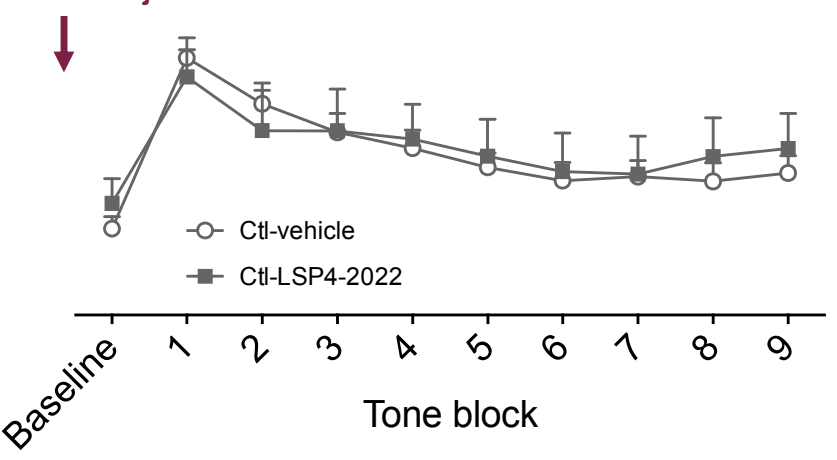

vehicle inj.

$\downarrow$

Figure 2 

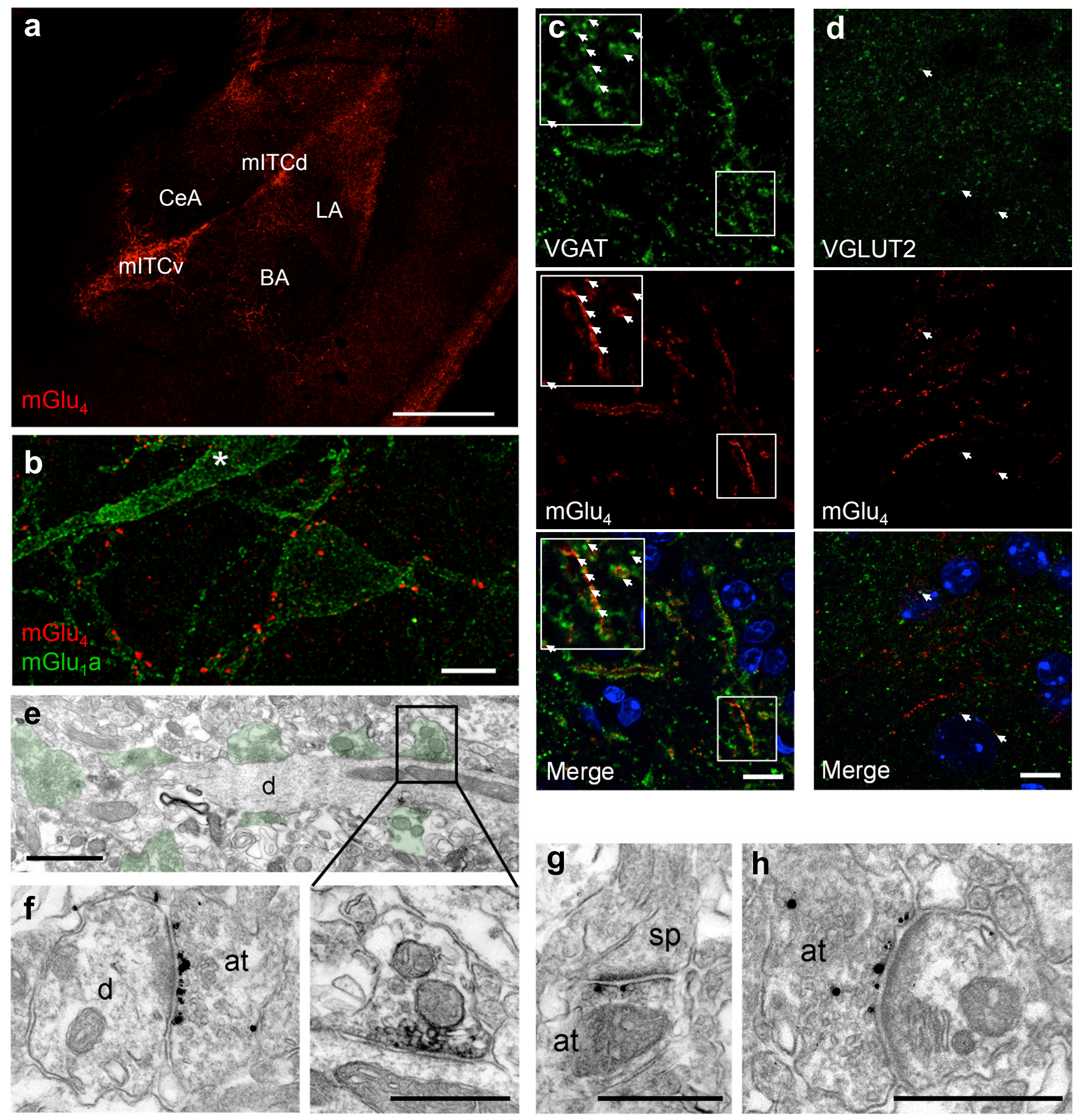

Figure 3 


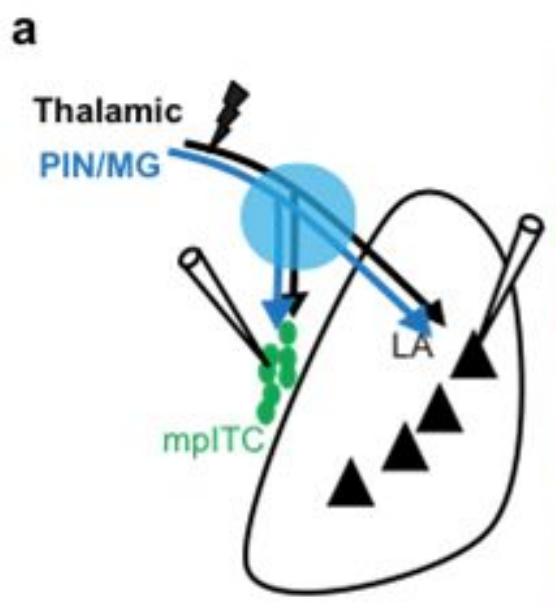

d

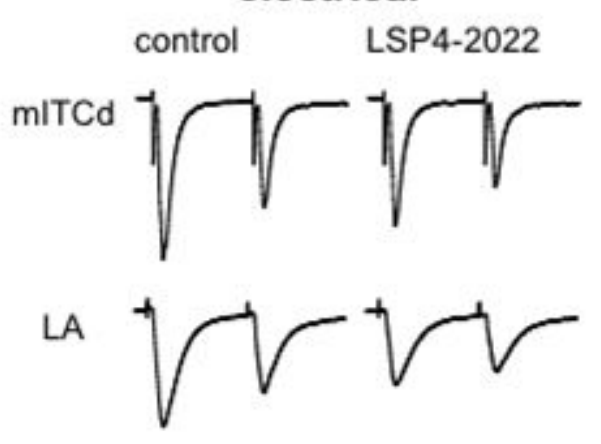

g

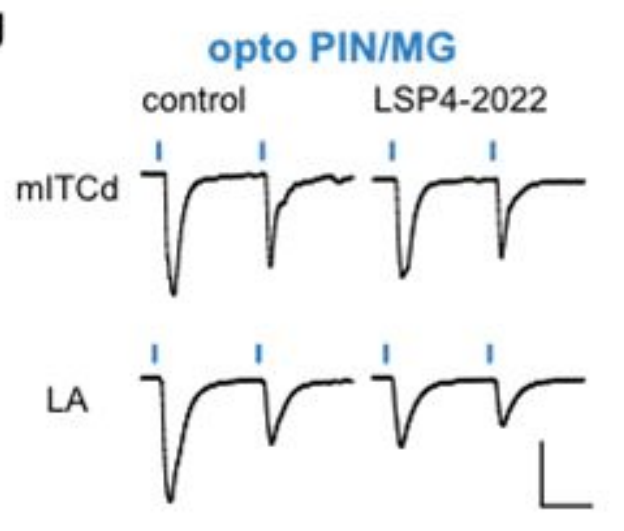

b

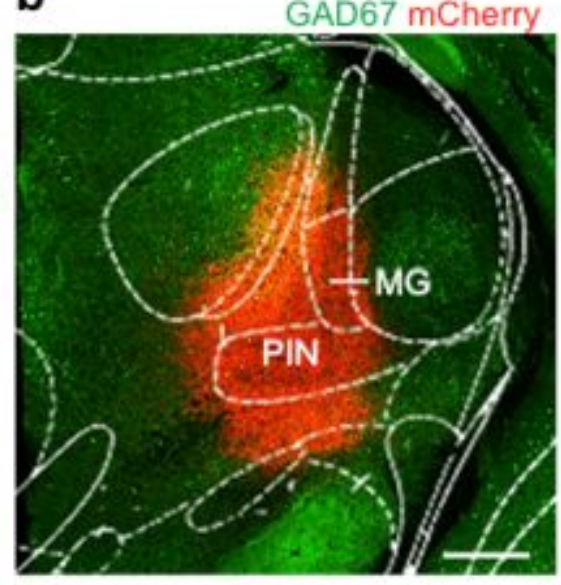

e

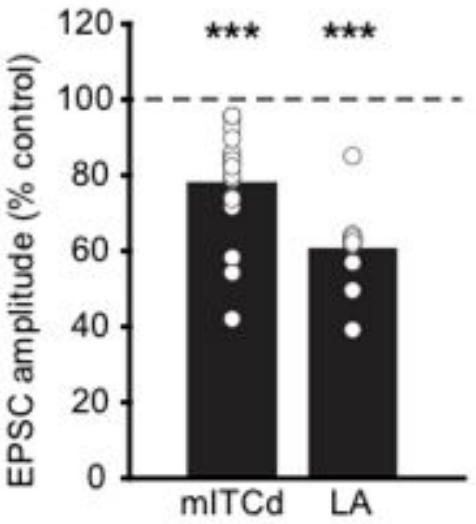

h

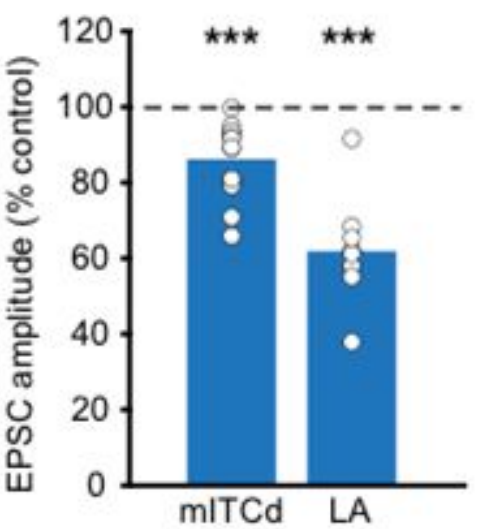

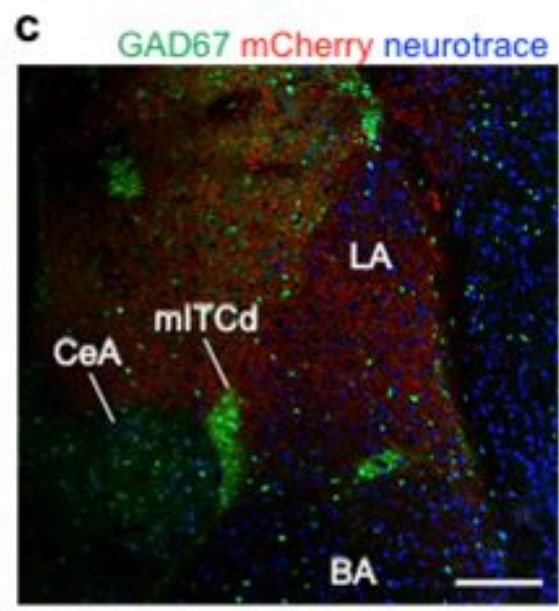

f

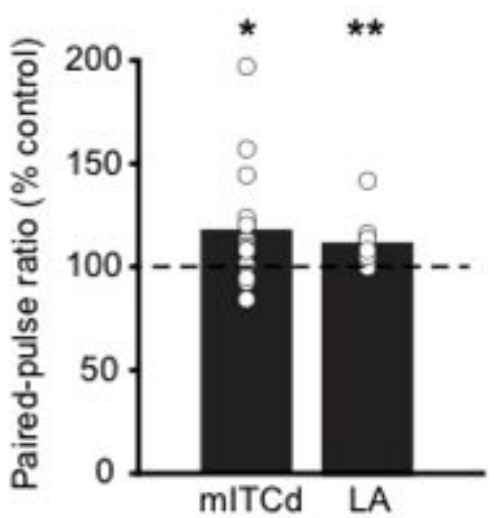

i

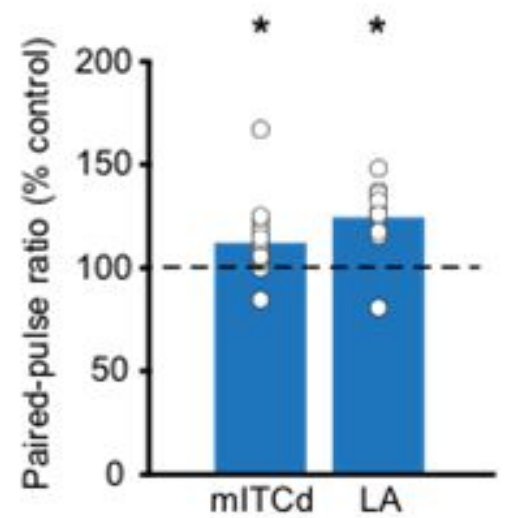


a<smiles>COc1cc(NC(=O)c2ccccn2)ccc1N=Nc1ccccc1Cl</smiles>

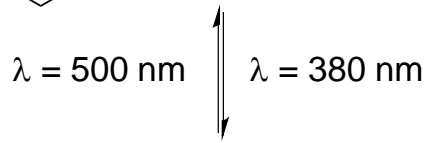

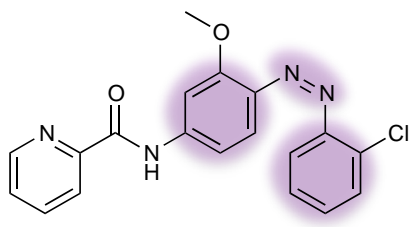

d

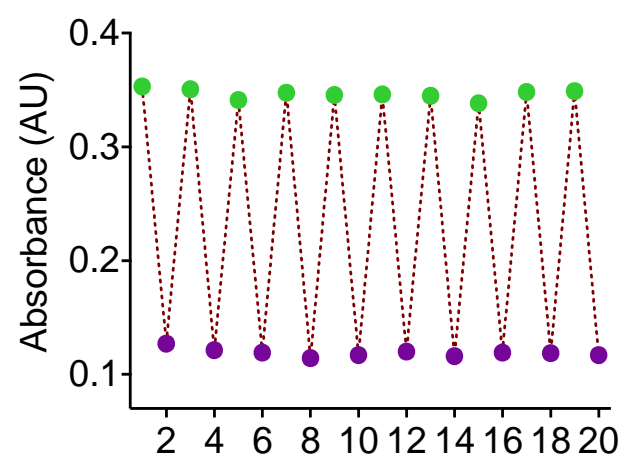

Cycle

g

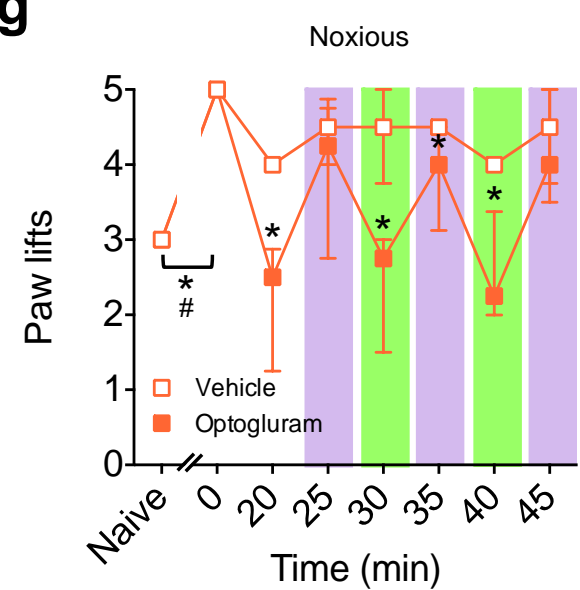

b

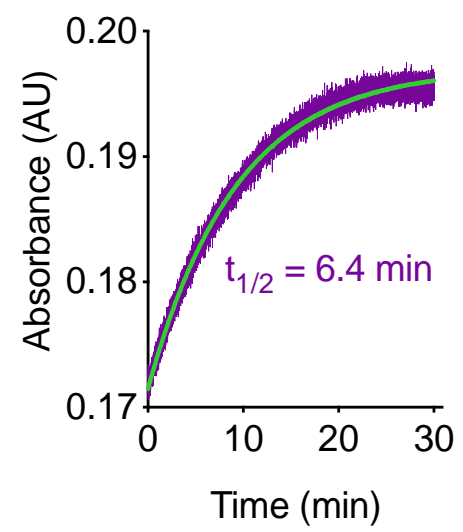

e

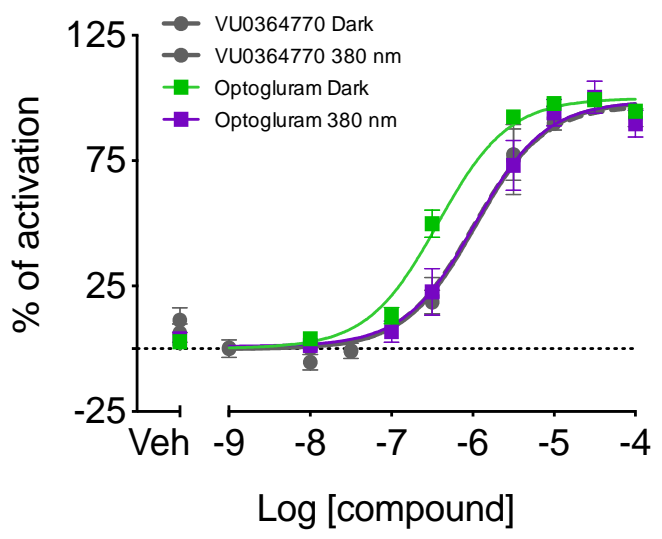

C

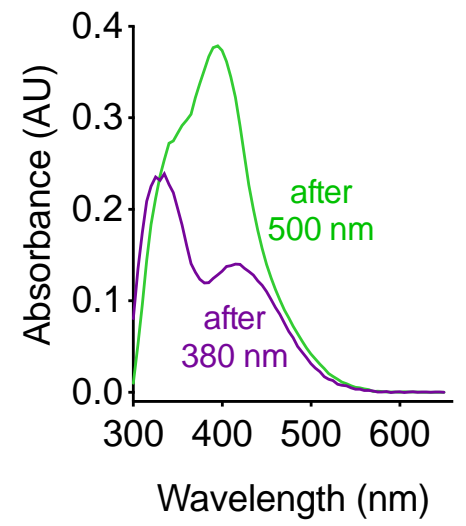

f

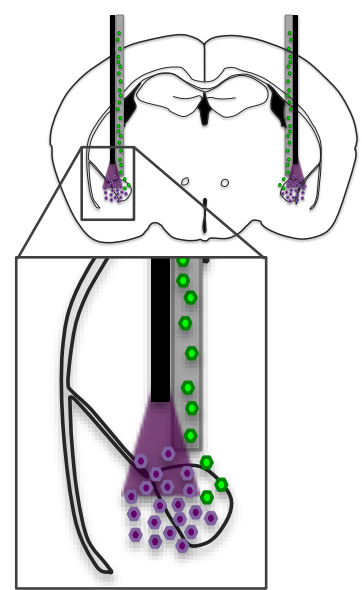

h

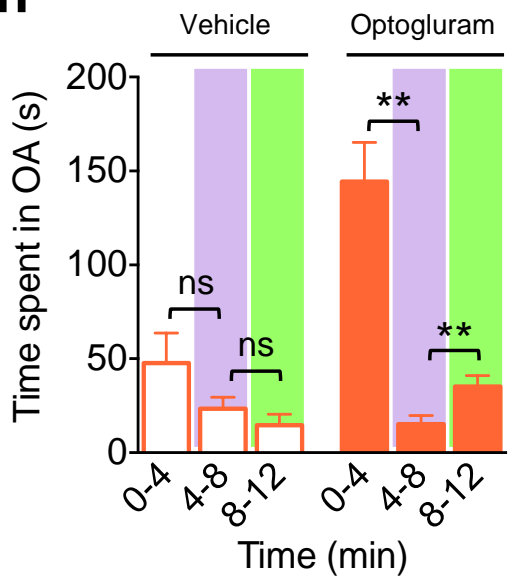

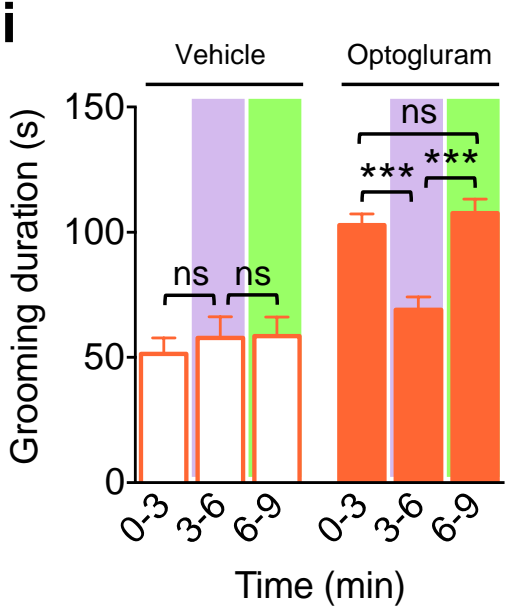

Figure 5 